\title{
Utilization patterns of Chinese medicine and Western medicine under the National Health Insurance Program in Taiwan, a population-based study from 1997 to 2003
} Lee-Chin Chang1,4, Nicole Huang2, Yiing-Jenq Chou ${ }^{2}$, Chen-Hua Lee ${ }^{3}$, Feng$\mathrm{Yu} \mathrm{Kao}{ }^{3}$ and Yi-Tsau Huang*1

Address: ${ }^{1}$ Institute of Traditional Medicine \& Institute of Public Health, School of Medicine, National Yang Ming University, Taipei, Taiwan, ${ }^{2}$ Department of Social Medicine \& Institute of Public Health, School of Medicine, National Yang Ming University, Taipei, Taiwan, ${ }^{3}$ Bureau of National Health Insurance, Taipei, Taiwan and ${ }^{4}$ Committee on Chinese Medicine and Pharmacy, Department of Health, Taipei, Taiwan

Email: Lee-Chin Chang - Lchin@ccmp.gov.tw; Nicole Huang - syhuang@ym.edu.tw; Yiing-Jenq Chou - yjchou@ym.edu.tw; ChenHua Lee - chlee@mail.nhi.gov.tw; Feng-Yu Kao - vaughn@mail.nhi.gov.tw; Yi-Tsau Huang* - huangyt@ym.edu.tw

* Corresponding author

Published: 9 August 2008

BMC Health Services Research 2008, 8:170 doi:10.1/86/1472-6963-8-170
Received: 24 July 2007

Accepted: 9 August 2008

This article is available from: http://www.biomedcentral.com/1472-6963/8/170

(c) 2008 Chang et al; licensee BioMed Central Ltd.

This is an Open Access article distributed under the terms of the Creative Commons Attribution License (http://creativecommons.org/licenses/by/2.0), which permits unrestricted use, distribution, and reproduction in any medium, provided the original work is properly cited.

\begin{abstract}
Background: In 1995, Taiwan has launched a national health-care system (the National Health Insurance Program, NHI) covering the use of both Western medicine (WM) and Chinese medicine (CM). This populationbased study was conducted to understand the role of $\mathrm{CM}$ in this dual medical system by determining the utilization patterns of CM and WM and to analyze the demographic characteristics and primary indications influencing the choice of the medical services for the development of strategies to enhance the appropriate use and reduce unnecessary use of $\mathrm{CM}$.
\end{abstract}

Methods: This study used the NHI sample files from 1997 to 2003 consisting of comprehensive utilization and enrolment information for a random sample of 200,432 NHI beneficiaries of the total enrolees from 1995 to 2000. A total of 136,720 subjects with valid and complete enrolment and utilization data were included in this study. The logistic regression method was employed to estimate the odds ratios (ORs) for utilization of CM and WM. The usage, frequency of services, and primary indications for $\mathrm{CM}$ and WM were evaluated. A significance level of $\alpha=0.05$ was selected.

Results: Compared with WM, the odds of CM increased from 1997 to 2003. The odds of using CM (OR = I.48; $95 \% \mathrm{Cl}:$ I.45-I.50; $\mathrm{p}<0.00 \mathrm{I})$ and WM $(\mathrm{OR}=1.74 ; 95 \% \mathrm{Cl}$ : I.72-I.77; $\mathrm{p}<0.00 \mathrm{I})$ were higher in females and that of $C M$ increased with age to a peak in the 45-54-year-group $(O R=1.75 ; 95 \% \mathrm{Cl}: 1.68-1.82 ; p<0.001)$ and WM $(O R=1.09 ; 95 \% \mathrm{Cl}: 1.05-1.13 ; p<0.00 \mathrm{I})$ in the elderly subjects ( $\geq 65$ years). The odds of CM and WM were similar in all income groups. However, those of CM were higher in Central (OR = 1.65; 95\% Cl: 1.56-1.74; p < $0.00 \mathrm{I})$ and Southern Taiwan (OR $=1.18 ; 95 \% \mathrm{Cl}: \mathrm{I} .12-1.25 ; \mathrm{p}<0.00 \mathrm{I})$ and lower in the remote areas $(\mathrm{OR}=$ $0.57 ; 95 \% \mathrm{Cl}: 0.52-0.63 ; \mathrm{p}<0.00 \mathrm{I}$ ). Most of the patients had one ambulatory visit of both medical services annually. However, the utilization of WM predominated over CM. Over $90 \%$ of CM service was provided by clinics, whereas over $60 \%$ of WM service by hospitals. Diseases of the respiratory system was the most frequent primary indication in CM and WM. Herbal medication was the most commonly used form of CM (68.4-72.7\%).

Conclusion: In recent years, there is an increasing trend in the utilization of CM in Taiwan. This increasing trend may be due to the covering of CM in the national health insurance system. 


\section{Background}

There is an increasing trend in the utilization of complementary or alternative medicine (CAM) worldwide [1]. According to the estimation of the World Health Organization, the usage of CAM ranges from 9 to $65 \%$ in different countries [2]. In the United States, CAM utilization increased from $34 \%$ in 1990 to $42 \%$ in 1997 [3,4]. CAM is also very popular in Europe [5], Canada [6], and Australia [7]. Patients choosing CAM are not necessarily dissatisfied with conventional medical care. They are seeking more effective ways to improve their health and wellbeing and to relieve symptoms associated with chronic, even terminal, illnesses or the side effects of conventional treatments for them [8].

Among different forms of CAM, Chinese medicine (CM) is well known for the medicinal formulas and acupuncture, and is one of the most popular alternative medicines in many countries. In Singapore, CM is the most popular form of CAM and has been employed by $88 \%$ of the CAM users [9]. Moreover, CM accounts for $40 \%$ of the health care in China [10]. Although CM is commonly used in the countries of East Asia, this system of medical service is also growing in popularity and offers an important alternative or complement to biomedical care in the Western countries [11].

In Taiwan, one distinguishing feature of the national health-care insurance system is the co-existence of the modern Western medicine (WM) and CM. In addition, the social health-care insurance system covers CM since 1975. Although only bone fractures and dislocations were included in the initial coverage of the Labor Medical Insurance, the therapeutic items were then expanded to internal medicine, gynecology, and acupuncture in 1983. In 1988, the Public Employee Medical Insurance Program started to reimburse CM [12]. After the implementation of the National Health Insurance (NHI) Program in March 1995, all citizens who have established a registered domicile in Taiwan are mandated to join the program. In June 2003, more than $99 \%$ of the 23-million population has benefited from this universal health insurance program. However, CM has only 5\% of the total expenditure in the NHI coverage. The health care items covered physician consultation for diagnosis, drug prescription, acupuncture, and muscle strain therapy [13].

Although information on different aspects of CM in Taiwan is available $[12,14-18]$, these reports were based on small samples $[12,14-16]$ or limited to one component of $\mathrm{CM}[17,18]$. Moreover, some of these studies were conducted before the establishment of the NHI program $[12,14,15]$. Recently, a study on the use frequencies, characteristics of users, and the disease categories treated by $\mathrm{CM}$ in Taiwan was conducted based on the complete data- sets of CM outpatient reimbursement claims from 1996 to 2001 [19]. However, except sex and age, other user characteristics were not analyzed and the utilization pattern of WM was only slightly explored. The purpose of this study is to gain a more complete picture of utilization of CM in the national health insurance system with dual medical systems (Western and Chinese medical services) by determining the extent of CM utilization from 1997 to 2003 using WM as a reference and the demographic factors and primary indications that predict the choice of the type of medical service.

\section{Methods \\ Data Resource}

Since all citizens in Taiwan who have established a registered domicile should be enrolled in the NHI Program, the NHI Bureau has accumulated 23.75 million administrative and claims records, forming the largest such collection in the world. The NHI research database was established under the cooperation of National Health Research Institutes (NHRI) and the NHI Bureau [13]. The NHRI safeguards the privacy and confidentiality of the subjects [20]. The database includes the NHI claims data and data from the enrolment and provider files. In addition to birth date and sex of each patient, the NHI claims data also record diagnosis, date of service, drugs prescribed and filed, dispensing method and anonymous identifiers for the patient, the hospital/clinic and the physician providing the service. All the individuals included in the entire claims database (the general population) were given different random numbers by using a random number function. Simple random sampling of about 50,000 people at a time was performed in 2000 . This sampling database consisted of four simple randomly sampled subsets and finally included 200,432 enrolees and represents $1 \%$ of the total NHI beneficiaries. [20,21].

\section{Study Sample}

In this study, a nationally representative sample of 200,432 NHI enrollees was used. To construct a fixed cohort, we excluded those who were newborns aged below 2 years $(5.0 \%)$ in 1997, died during the study period $(3.4 \%)$, were foreigners $(4.1 \%)$, had incomplete data $(1.0 \%)$, and had no continuous enrollment information from 1997 to 2003 (18.2\%) sequentially. The final fixed cohort consisted of 136,720 individuals. These individuals were observed to investigate the longitudinal utilization patterns of CM and WM from 1997 to 2003.

\section{Study variables}

In order to understand the common factors affecting the utilization of CM and WM, we selected the demographic factors according to previous studies [12,22,23]. In addition to gender, ages were categorized into eight groups: 2$7,8-14,15-24,25-34,35-44,45-54,55-64$ and 65 
years or older. For the socio-economic status (SES), we classified those with a well-defined monthly wage into three categories: $\geq$ US\$1,280, US\$640-1,279 and < US $\$ 640$. Those people without a well-defined monthly wage were categorized into two groups: farmers and fishermen, and others, which include veterans, low-income people and individuals enrolled in the NHI through local government offices. Severe diseases were defined according to the Illness and Injury Severity Score and the NHI has an official list of major diseases including cancer, acquired immune deficiency syndrome, major psychiatric disorder, and so on [13]. Remote areas were defined officially according to mountainous geographic environment and traffic conditions. There are 30 mountainous regions proclaimed remote areas [21]. In addition, offshore islands include Lanyu Isles, Green Island, and Penghu Islands. These remote areas have been under-served with insufficient medical services.

\section{Definition of CM}

In this study, CM is used pragmatically to refer to diagnostic and therapeutic practices, including primarily herbal medication, acupuncture, and muscle strain therapy. Muscle strain therapy includes professional massage and osteopathy manual therapy. In this study, we also considered dislocation treatment as one component of the muscle strain therapy. These three components of $\mathrm{CM}$ are also modalities of CAM defined in the Western countries [3]. In addition to these three main components, CM users may also consult the physician only for physical examination or suggestion of management of the health care problems but without treatment. Consultation services were also covered by the NHI Program.

\section{Statistical analysis}

The unit of observation was each individual in the study sample. The usage, frequency of services, and primary indications for CM and WM were evaluated. The classification of primary indications was according to the International Classification of Diseases Ninth Clinical Modification (ICD-9-CM) [25]. The statistical software SAS 9.13 [26] was used for data management and analyses. A logistic regression model was used to analyze the data, and generalized estimating equations were used to account for correlation among the repeated measurements $[27,28]$. Based on the correlation matrices of CM and WM utilizations over time, an unstructured working correlation was selected. In addition, sensitivity analyses were conducted for autoregressive, independent and compound symmetry, and the results remained robust. A significance level of $\alpha=0.05$ was selected.

\section{Results}

Table 1 shows the demographic characteristics of the studied cohort. Adjusted ORs and 95\% confidence intervals
Table I: Demographic characteristics of the studied cohort $(n=$ 136,720)

\begin{tabular}{|c|c|c|}
\hline Characteristic* & No. of subjects & $\%$ \\
\hline \multicolumn{3}{|l|}{ Gender } \\
\hline Male & 68,135 & 49.8 \\
\hline Female & 68,585 & 50.2 \\
\hline \multicolumn{3}{|l|}{ Age (years) } \\
\hline $2-7$ & 9,648 & 7.1 \\
\hline $8-14$ & 11,933 & 8.7 \\
\hline $15-24$ & 23,244 & 17.0 \\
\hline $25-34$ & 26,903 & 19.7 \\
\hline $35-44$ & 27,307 & 20.0 \\
\hline $45-54$ & 17,273 & 12.6 \\
\hline $55-64$ & 9,986 & 7.3 \\
\hline$\geq 65$ & 10,426 & 7.6 \\
\hline \multicolumn{3}{|l|}{ Socio-economic status } \\
\hline$<$ US\$640 & 54,729 & 40.0 \\
\hline US\$640-I 279 & 29,788 & 21.8 \\
\hline$\geq$ US $\$ 1280$ & 11,137 & 8.1 \\
\hline Farmers and fishermen & 21,568 & 15.8 \\
\hline Others & 19,498 & 14.3 \\
\hline \multicolumn{3}{|l|}{ Severe disease } \\
\hline Without & 132,835 & 97.2 \\
\hline With & 3,885 & 2.8 \\
\hline \multicolumn{3}{|l|}{ Region } \\
\hline Taipei & 43,392 & 31.7 \\
\hline Northern Taiwan & 18,830 & 13.8 \\
\hline Central Taiwan & 26,732 & 19.6 \\
\hline Southern Taiwan & $21,24 \mid$ & 15.5 \\
\hline Kaohsiung and Pingtung & 22,848 & 16.7 \\
\hline East Taiwan & 3,677 & 2.7 \\
\hline \multicolumn{3}{|l|}{ Remote Area } \\
\hline Mountainous regions & 1,159 & 0.9 \\
\hline Offshore islands & 1,156 & 0.9 \\
\hline General Population & 134,405 & 98.2 \\
\hline
\end{tabular}

*Determined from 1997 prospectively.

(95\% CIs) resulting from the logistic regression model are displayed in Table 2. The odds of using CM (OR 1.48) and WM (OR 1.74) were higher for females than for males (OR 1.00). Compared with the youngest age group (OR $1.00)$, the odds of CM increased with age to a peak in the 45-54-year-group (OR 1.75) whereas those of WM became relatively high only in the elderly subjects $(\geq 65$ years) (OR 1.09).

The odds of CM (OR 1.05) and WM (OR 1.00) in the group with a monthly wage US\$640-1,279 were higher than those in the low-income group ( $<$ US\$640) (CM: OR 1.04; WM: OR 0.98), farmers and fishermen (CM: OR: 0.90; WM: OR 0.97) and the other group (CM: OR 0.86; WM: OR: 0.77). Although the odds of CM were similar in the groups with (OR 1.00) and without (OR 1.00) severe diseases, the odds of WM in the people with severe diseases (OR 2.40) was higher than those without these diseases (OR 1.00) (Table 2). 
Table 2: Adjusted odds ratios and $95 \%$ confidence intervals for characteristics associated with the utilization of Chinese medicine and Western medicine $(n=136,720)$

\begin{tabular}{|c|c|c|c|c|}
\hline Characteristic & Chinese medicine & $p$ value & Western medicine & $p$ value \\
\hline \multicolumn{5}{|l|}{ Gender } \\
\hline Male & 1.00 & & 1.00 & \\
\hline Female & $1.48(\mathrm{I} .45-1.50)^{*}$ & $<0.001$ & $1.74(1.72-1.77)$ & $<0.001$ \\
\hline \multicolumn{5}{|l|}{ Age (years) } \\
\hline $2-7$ & 1.00 & & 1.00 & \\
\hline $8-14$ & $1.14(1.10-1.18)$ & $<0.001$ & $0.50(0.49-0.5 \mathrm{I})$ & $<0.001$ \\
\hline $15-24$ & $1.38(1.33-1.44)$ & $<0.001$ & $0.36(0.36-0.37)$ & $<0.001$ \\
\hline $25-34$ & $1.58(1.52-1.64)$ & $<0.001$ & $0.38(0.37-0.38)$ & $<0.001$ \\
\hline $35-44$ & $1.74(1.67-1.81)$ & $<0.001$ & $0.39(0.38-0.40)$ & $<0.001$ \\
\hline $45-54$ & $1.75(1.68-1.82)$ & $<0.001$ & $0.45(0.44-0.46)$ & $<0.001$ \\
\hline $55-64$ & $1.63(1.56-1.71)$ & $<0.001$ & $0.64(0.63-0.66)$ & $<0.001$ \\
\hline$\geq 65$ & $1.51(1.44-1.58)$ & $<0.001$ & $1.09(1.05-1.13)$ & $<0.001$ \\
\hline \multicolumn{5}{|l|}{ Socioeconomic status } \\
\hline$\geq$ US $\$ 1,280$ & 1.00 & & 1.00 & \\
\hline US\$640-I,279 & $1.05(1.03-1.07)$ & $<0.001$ & $1.00(0.98-1.01)$ & 0.473 \\
\hline$<$ US $\$ 640$ & $1.04(1.02-1.06)$ & $<0.001$ & $0.98(0.96-0.99)$ & $<0.001$ \\
\hline Farmers and fishermen & $0.90(0.87-0.92)$ & $<0.001$ & $0.97(0.95-0.99)$ & $<0.001$ \\
\hline Others & $0.86(0.84-0.88)$ & $<0.001$ & $0.77(0.76-0.78)$ & $<0.001$ \\
\hline \multicolumn{5}{|l|}{ Severe disease } \\
\hline Without & 1.00 & & 1.00 & \\
\hline With & $1.00(0.95-1.05)$ & 0.900 & $2.40(2.29-2.52)$ & $<0.001$ \\
\hline \multicolumn{5}{|l|}{ Region } \\
\hline Eastern Taiwan & 1.00 & & 1.00 & \\
\hline Taipei & $0.91(0.86-0.96)$ & 0.001 & $1.03(0.99-1.07)$ & 0.117 \\
\hline Northern Taiwan & $0.96(0.90-1.01)$ & 0.123 & $1.08(1.04-1.13)$ & $<0.001$ \\
\hline Central Taiwan & $1.65(1.56-1.74)$ & $<0.001$ & $1.15(1.11-1.20)$ & $<0.001$ \\
\hline Southern Taiwan & $1.18(1.12-1.25)$ & $<0.001$ & $1.20(1.15-1.25)$ & $<0.001$ \\
\hline Kaohsiung and Pingtung & $1.09(1.03-1.16)$ & 0.002 & $1.21(1.16-1.26)$ & $<0.001$ \\
\hline \multicolumn{5}{|l|}{ Remote Area } \\
\hline General Population & 1.00 & & 1.00 & \\
\hline Mountainous regions & $0.57(0.52-0.63)$ & $<0.001$ & $1.03(0.96-1.11)$ & 0.356 \\
\hline Offshore islands & $0.78(0.70-0.86)$ & $<0.001$ & $1.21(1.13-1.30)$ & $<0.001$ \\
\hline \multicolumn{5}{|l|}{ Season } \\
\hline Winter (December - February) & 1.00 & & 1.00 & \\
\hline Spring (March - May) & $0.95(0.95-0.96)$ & $<0.001$ & $0.88(0.88-0.89)$ & $<0.001$ \\
\hline Summer (June - August) & $0.90(0.89-0.90)$ & $<0.001$ & $0.79(0.79-0.80)$ & $<0.001$ \\
\hline Autumn (September - November) & $0.93(0.93-0.94)$ & $<0.001$ & $0.86(0.85-0.86)$ & $<0.001$ \\
\hline \multicolumn{5}{|l|}{ Year } \\
\hline 1997 & 1.00 & & 1.00 & \\
\hline 1998 & $1.05(1.03-1.06)$ & $<0.001$ & $1.10(1.10-1.11)$ & $<0.001$ \\
\hline 1999 & $1.10(1.09-1.12)$ & $<0.001$ & $1.21(1.20-1.22)$ & $<0.001$ \\
\hline 2000 & $1.10(1.09-1.11)$ & $<0.001$ & $1.26(1.25-1.27)$ & $<0.001$ \\
\hline 2001 & $1.11(1.09-1.12)$ & $<0.001$ & $1.27(1.26-1.28)$ & $<0.001$ \\
\hline 2002 & $1.12(1.10-1.13)$ & $<0.001$ & $1.28(1.26-1.29)$ & $<0.001$ \\
\hline 2003 & $1.15(1.14-1.17)$ & $<0.001$ & $1.17(1.16-1.19)$ & $<0.001$ \\
\hline
\end{tabular}

*Odds ratio ( $95 \%$ confidence interval).

The odds of CM were higher among people in Central (OR 1.65), Southern (OR 1.18), and Kaohsiung and Pingtung (OR 1.09) than those in Eastern Taiwan (OR 1.00), Northern Taiwan (OR 0.96), and Taipei (OR 0.91). However, those of WM were higher among people in Taipei (OR 1.03), Northern Taiwan (OR 1.08), Central Taiwan (OR 1.15), Southern Taiwan (OR 1.20), and Kaohsiung and Pingtung (OR 1.21) than Eastern Taiwan (OR 1.00). The odds of CM in the general population (OR 1.00) was higher than those in the mountainous regions (OR 0.57) and offshore islands (OR 0.78) whereas those of WM were higher in mountainous regions (OR 1.03) and offshore islands (OR 1.21) than the general population (OR 1.00) (Table 2).

Table 2 also shows a steady increasing trend in the odds of CM from 1997 (OR 1.00) to 2003 (OR 1.15). Moreover, the odds WM utilization also increased from 1997 (OR 
1.00 ) to 2002 (OR 1.28) but decreased in 2003 (OR 1.17). Of all seasons, the odds of either CM or WM were the highest in winter.

The crude utilizations of CM and WM are shown in Table 3. The number of CM users increased from 36,372 in 1997 to 41,823 in 2003. However, the number of WM users from 115,833 in 1997 to 121,605 in 2002 and decreased to 120,926 in 2003 . The average number of patients who had used any CM service in one year and the number of visits to CM providers were 39,562 and 191,612, respectively. The corresponding figures for WM were 119,915 and $1,542,342$. Most of the patients had one ambulatory visit a year. However, the utilization frequency of WM $($ mean $=12.55-13.34$, median $=8-9)$ was higher than that of CM (mean $=4.63-5.03$, median =2-3). Most of the ambulatory CM service was provided by clinics (93.4$96.1 \%$ ) and only 3.9-6.6\% was provided by hospitals. Moreover, the frequency of ambulatory visits in hospitals steadily increased from $3.9 \%$ in 1997 to $6.6 \%$ in 2002 . On the other hand, clinics provided about two-thirds (60.5$67.8 \%$ ) of the ambulatory WM service, outpatient department of the hospitals took the remaining one-third (32.2$39.5 \%$ ) of ambulatory service.

Diseases of the respiratory system (CM 22.1\%, WM $35.6 \%)$ and the musculoskeletal system and connective tissue (CM 18.1\%, WM 7.5\%) were the top two primary indications in the ambulatory health care of both $\mathrm{CM}$ and WM. In CM, the remaining common primary indications were injury and poisoning (16.2\%), diseases of signs, symptoms and ill-defined conditions (14.2\%), and diseases of the digestive system (11.4\%). In WM, diseases of the genitourinary system (7.2\%), diseases of the digestive system (7.2\%), and diseases of sense organs (7.1\%) were also common primary indications (Table 4$)$.

Herbal medication was the most important component of $\mathrm{CM}$. This component accounted for more than two-thirds of the ambulatory visits (68.4-72.7\%). The utilization rate decreased from $70.8 \%$ in 1997 to $68.7 \%$ in 2003 . The other two major components were muscle strain therapy (including dislocation therapy) and acupuncture. Muscle strain therapy accounted for $15.6-17.5 \%$ and acupuncture for $9.2-13.0 \%$ of the ambulatory visits. These two components showed increasing utilization trends: muscle strain therapy increased from $16.4 \%$ in 1997 to $17.2 \%$ in 2003 and acupuncture from $9.4 \%$ in 1997 to $13.0 \%$ in 2003. In addition to these main components of CM, general consultation services accounted for 3.4\% in 1997. However, the utilization of these consultation only services decreased to $1.1 \%$ in 2003 (Table 5).

\section{Discussion}

In this study, the logistic regression method was used to identify patient characteristics associated with the utilization patterns of CM and WM over time. Females used both health care services more than males. The higher utilization of CM in females has also been reported in Singapore [9] and the Western countries [11,29-32] as well as in Taiwan $[12,14,16,19]$. In practice, CAM has been used to treat postpartum conditions, menopause, and chronic diseases among women [16,19,29,32-35]. Moreover, the age distribution of CM utilization peaked at 45-54 years. This finding is similar to those reported previously $[11,12,16,19,29-32]$. Since WM provides health-care services such as vaccinations for the children [36], the utilization was revealed to increase with age and peak in

Table 3: Crude utilizations of Chinese and Western medicine under the NHI program, 1997-2003 (total population = 136,720)

\begin{tabular}{|c|c|c|c|c|c|c|c|}
\hline \multirow[b]{2}{*}{ Year } & \multirow[b]{2}{*}{ No. of users } & \multirow[b]{2}{*}{ No. of visits } & \multicolumn{3}{|c|}{ Frequency of utilization } & \multicolumn{2}{|c|}{ Provider } \\
\hline & & & Mean \pm SD & Median & Mode & Hospital (\%) & Clinic (\%) \\
\hline \multicolumn{8}{|c|}{ Chinese medicine } \\
\hline 1997 & 36,372 & 181,109 & $4.98 \pm 6.59$ & 3 & I & $7,060(3.9)$ & I 74,049 (96.I) \\
\hline 1998 & 37,622 & 189,330 & $5.03 \pm 6.60$ & 3 & 1 & $7,709(4.1)$ & $|81,62|(95.9)$ \\
\hline 1999 & 39,635 & 196,908 & $4.97 \pm 6.36$ & 3 & I & $9,461(4.8)$ & 187,447 (95.2) \\
\hline 2000 & 40,227 & 188,713 & $4.69 \pm 5.81$ & 2 & I & $10,330(5.5)$ & $178,383(94.5)$ \\
\hline 2001 & 40,425 & 187,198 & $4.63 \pm 5.73$ & 3 & I & $10,865(5.8)$ & $176,333(94.2)$ \\
\hline 2002 & 40,833 & 191,251 & $4.68 \pm 5.82$ & 3 & I & $12,617(6.6)$ & $178,634(93.4)$ \\
\hline 2003 & 41,823 & 206,777 & $4.94 \pm 6.45$ & 3 & I & II,497 (5.6) & $195,280(94.4)$ \\
\hline \multicolumn{8}{|c|}{ Western medicine } \\
\hline 1997 & 115,833 & $\mathrm{I}, 486,570$ & $12.83 \pm 13.82$ & 8 & 1 & 478,493 (32.2) & I,008,077 (67.8) \\
\hline 1998 & $1 \mid 8,504$ & $\mathrm{I}, 553,383$ & $|3.1| \pm \mid 4.07$ & 9 & 1 & $509,743(32.8)$ & $1,043,640(67.2)$ \\
\hline 1999 & 120,362 & $1,606,111$ & $13.34 \pm 13.85$ & 9 & 1 & $549,420(34.2)$ & $|, 056,69|(65.8)$ \\
\hline 2000 & 120,982 & I,552,77| & $12.83 \pm 12.92$ & 9 & I & $554,656(35.7)$ & $998,115(64.3)$ \\
\hline 2001 & 121,190 & $\mathrm{I}, 533,878$ & $12.66 \pm 12.97$ & 9 & I & $583,055(38.0)$ & $950,823(62.0)$ \\
\hline 2002 & 121,605 & $\mathrm{I}, 545,956$ & $12.71 \pm 13.23$ & 9 & I & $610,399(39.5)$ & $935,557(60.5)$ \\
\hline 2003 & 120,926 & $\mathrm{I}, 5 \mathrm{I} 7,722$ & $12.55 \pm 13.69$ & 8 & I & $568,128(37.4)$ & $949,594(62.6)$ \\
\hline
\end{tabular}


Table 4: Primary indications in ambulatory visits of Chinese and Western medicine under the National Health Insurance Program in Taiwan from 1997 to 2003

\begin{tabular}{|c|c|c|}
\hline Primary indication (ICD-9-CM code) & $\begin{array}{c}\text { Chinese medicine } \\
(n=I, 34 I, 286)\end{array}$ & $\begin{array}{l}\text { Western medicine } \\
(n=10,796,391)\end{array}$ \\
\hline I Infectious and parasitic diseases & $0.5^{*}$ & 2.3 \\
\hline 2 Malignant neoplasms & 0.2 & 0.7 \\
\hline 3 Other neoplasms & 0.1 & 0.7 \\
\hline 4 Endocrine, nutritional and metabolic diseases and immunity disorders & 1.3 & 3.8 \\
\hline 5 Mental disorders & 0.6 & 2.0 \\
\hline 6 Diseases of the nervous system & 1.6 & 1.3 \\
\hline 7 Diseases of the sense organs & 1.2 & 7.1 \\
\hline 8 Diseases of the circulatory system & 1.8 & 6.3 \\
\hline 9 Diseases of the respiratory system & 22.1 & 35.6 \\
\hline 10 Diseases of the digestive system & 11.4 & 7.2 \\
\hline II Diseases of the genitourinary system & 7.2 & 7.2 \\
\hline I2 Complications of pregnancy, childbirth and the puerperium & 0.1 & 0.4 \\
\hline I3 Diseases of skin and subcutaneous tissue & 3.1 & 6.2 \\
\hline I4 Diseases of the musculoskeletal system and connective tissue & 18.1 & 7.5 \\
\hline I5 Congenital anomalies & 0.1 & 0.1 \\
\hline 16 Certain conditions originating in the perinatal period & 0.0 & 0.0 \\
\hline 17 Signs, symptoms and ill-defined conditions & 14.2 & 3.7 \\
\hline I 8 Injury and poisoning & 16.2 & 4.5 \\
\hline 19 Accidents and self inflicted injury & 0.0 & 0.1 \\
\hline 20 Other reasons for contact with health services & 0.2 & 3.3 \\
\hline
\end{tabular}

$* \%$.

youngest group (2-7 years). Moreover, WM utilization was highest in the elderly people ( $\geq 65$ years).

The utilization of CM was higher in the regular salary income group than the low-income group, farmers and fishermen and the other group. These results are similar to the previous findings that CAM users are those with higher education and in the middle to upper socioeconomic status $[3,4,37,38]$. WM was more frequently used by patients with severe diseases. These diseases require long-term supportive treatment and may not be suitable for CM. Although CM usage was more prevalent in the central and southern parts of Taiwan, CM was not frequently used in mountainous areas and offshore islands. One possible explanation for this phenomenon is the uneven distribution of CM providers, since there are only $6 \mathrm{CM}$ hospitals and $24 \mathrm{CM}$ clinics in eastern Taiwan [13].

In a previous study, $62.5 \%$ of the valid beneficiaries in the NHI Program have been reported to use CM at least once from 1996 to 2001 [19]. By controlling the co-variant factors, we found an increasing trend in the utilization of $\mathrm{CM}$ and WM. Moreover, 39,562 patients used CM services in each year whereas the corresponding figures for WM were 119,915 . Most of patients had a single ambulatory visit annually. However, the mean utilization frequency of WM (12.55-13.34) was higher than that of CM (4.635.03). The numbers reported in this study may seem higher than those reported previously [19] because these values were based on only users, not total population. In addition, we revealed that over $90 \%$ of the CM services

Table 5: Percentage distribution of ambulatory visits for different components of Chinese medicine under the National Health Insurance Program in Taiwan from 1997 to 2003

\begin{tabular}{lccccccc}
\hline & \multicolumn{5}{c}{ Year } \\
\cline { 2 - 7 } Component* & 1997 & 1998 & 1999 & 2000 & 2001 & 2002 & 2003 \\
\hline Herbal medication & 70.8 & 72.7 & 71.8 & 70.5 & 69.3 & 68.4 & 68.7 \\
Muscle strain therapy (Including dislocation therapy) & 16.4 & 15.6 & 15.6 & 16.0 & 16.6 & 17.5 & 17.2 \\
Acupuncture & 9.4 & 9.2 & 10.0 & 11.5 & 12.1 & 12.9 & 13.0 \\
Consultation only and others & 3.4 & 2.5 & 2.6 & 2.0 & 2.0 & 1.2 & 1.1 \\
\hline Total & & & & & & & \\
& 179,464 & 205,637 & 221,440 & 206,108 & 208,487 & 210,720 & 225,705 \\
\hline
\end{tabular}

*More than one component may be provided in each visit. 
were provided by clinics whereas clinics only provided about two-thirds (60.5-67.8\%) of the ambulatory WM services. These findings were consistent with those reported previously $[15,19,39]$. Under the NHI Program, copayments vary with the provider type. They are highest for ambulatory health care at medical centres and lowest for clinics [40]. Moreover, the copayments in CM are lower than WM. Therefore, more than $90 \%$ of the CM users visited clinics and less than 10\% CM hospitals.

Based on the ICD-9-CM code, we found that diseases of the respiratory system, diseases of the musculoskeletal system and connective tissue, injury and poisoning, signs, symptoms and ill-defined conditions, and diseases of the digestive system were the primary indications in CM. Similar patterns have been reported previously $[12,14]$. However, the top two primary indications were also the same as those in WM. We have also found that the utilization of these two health-care services was higher in winter than in the remaining seasons. These findings may be due to the fact that diseases of the respiratory system occurs more frequency in winter. These findings indicate that $\mathrm{CM}$ may be a feasible complementary choice for the patients with respiratory diseases.

Herbal medication is the major component of $\mathrm{CM}$ accounting for more than two-thirds of the ambulatory visits. In the United States, herbal products have an annual market of US $\$ 5.1$ billion [41] and 38.2 million adults have used herbs and supplements [42]. The herbal prescriptions in the Western countries are usually with a single herb whereas the majority of prescriptions in Taiwan are composed of 3-6 herbs and are often prescribed for three times a day [17]. Under the NHI program, herbal formulas were provided as standard pharmaceutical products in standard powder forms. This strategy not only can control the quality of the prescriptions but also enable the standardization of herbal formulas. Acupuncture is another popular form of CAM [3-7]. It is mainly employed in the treatment of neurologic and musculoskeletal diseases $[18,43,44]$. In Taiwan, about $23 \%$ of people used acupuncture from 1996 to 2002 [18]. We obtained similar results in this study. In addition to an increasing trend in the utilization of acupuncture, the prevalence of muscle strain therapy was also increased in recent years. This increase may be due to the increase in the number of providers [13]. However, further investigations are needed to confirm this suggestion.

This study is the first population-based investigation to determine the utilization patterns of CM and WM in Taiwan under the NHI Program. Since we obtained the data from a sufficiently large national representative sample from the NHI sample files, some of the common shortcomings in interview or questionnaire surveys may be avoided. The large sample size and the comprehensive datasets allow us to study a wide array of factors in the general population. However, we have excluded enrolees who were newborns aged below 2 years in 1997, those died in the study period, aliens, those with incomplete data, and those without continuous enrolment information. This manipulation of the data may lead to some selection bias. Since we used only the NHI claims data, we are unable to determine the utilization of $\mathrm{CM}$ services not covered by the NHI program. Therefore, the utilization of $\mathrm{CM}$ in Taiwan might be underestimated.

\section{Conclusion}

The utilization of CAM has rapidly increased in many countries during the last two decades [4-7]. CAM is rarely covered in national health systems. However, $75 \%$ of the Dutch population wanted hospitals to provide CAM [45] and $74 \%$ of the people in UK felt that complementary therapies should be available on the national health system [46]. In Taiwan, the NHI Program is a comprehensive and universal health insurance program. This program not only covers conventional WM services, but also traditional CM services. Moreover, $\mathrm{CM}$ is popular and more than $60 \%$ of all beneficiaries of this health insurance system had used CM at least once a year [19]. In this study, we found that the rate of increase in the use of $\mathrm{CM}$ was smaller than that in WM (Table 2) and that the increase was not apparently shown in the number of visits per user. Taiwan's experience of covering CM services under its national health insurance system may serve as an important reference to other countries. This strategy offers people another choice for medical care services with mandated coverage.

\section{Competing interests}

The authors declare that they have no competing interests.

\section{Authors' contributions}

LCC, NH, YJC, CHL and YTH contributed equally to this paper. FYK carried out the analysis. LCC, NH, YJC, CHL, FYK and HYT pictured the idea and drafted the manuscript. All authors have read and approved the final manuscript.

\section{Acknowledgements}

This study is based on the data from the National Health Insurance Research Database provided by the Bureau of National Health Insurance and managed by the National Health Research Institutes in Taiwan. The interpretations and conclusions do not represent those of Bureau of National Health Insurance or National Health Research Institutes.

\section{References}

I. Goldbeck-Wood S, Dorozynski A, Lie LG: Complementary medicine is booming worldwide. $B M|1996,3| 3:|3|-\mid 33$.

2. Ernst E: Prevalence of use of complementary/alternative medicine: a systematic review. Bull WHO 2000, 78:252-257. 
3. Eisenberg DM, Kessler RC, Foster C: Unconventional medicine in the United States. N Engl J Med 1993, 328:246-252.

4. Eisenberg DM, Davis RB, Ettner SL, Appels S, Wilkey S, Van Rompay $M$, Kessler RC: Trends in alternative medicine use in the United States, 1990-1997. Results of a follow-up national survey. JAMA 1998, 280:1569-1575.

5. Fisher P, Ward W: Complementary medicine in Europe. BMJ 1994, 309:107-110.

6. Millar WJ: Use of alternative health care practitioners by Canadians. Can J Public Health 1997, 88: I54-I58.

7. MacLennan AH, Wilson DH, Taylor AW: Prevalence and cost of alternative medicine in Australia. Lancet 1996, 347:569-573.

8. Astin JA: Why patients use alternative medicine: results of a national study. JAMA 1998, 279: I548-I553.

9. Lim MK, Sadarangani P, Chan HL, Heng JY: Complementary and alternative medicine use in multiracial Singapore. Compl Therap Med 2005, 13:16-24.

10. World Health Organisation: Regional Strategy for Traditional Medicine in Western Pacific World Health Organization Western Pacific Manila; 2002.

II. Cassidy CM: Chinese medicine users in the United States. Part I: Utilization, satisfaction, medical plurality. J Altern Compl Med 1998, 4:17-27.

12. Chi C, Lee JL, Lai JS, Chen SC, Chen CY, Chang SK: Utilization of Chinese medicine in Taiwan. Altern Ther Health Med 1997, 3:40-53.

13. Bureau of National Health Insurance, Taiwan: National Health Insurance Profile, Taipei (last accessed 27 June 2008) 2004 [http:/l www.nhi.gov.tw].

14. Kang JT, Lee CF, Chen CF, Chou P: Factors related to the choice of clinic between Chinese traditional medicine and Western medicine. J Formosan Med Assoc 1994, 93:S49-S55.

15. Chi C, Lee JL, Lai JS, Chen CY, Chang SK, Chen SC: The practice of Chinese medicine in Taiwan. Soc Sci Med 1996, 43: I329-1348.

16. Chou P: Factors related to utilization of traditional Chinese medicine in Taiwan. Zhonghua Yi Xue Za Zhi (Taipei) 200I, 64(4): $19 \mid-202$.

17. Chen LC, Wang BR, Chou YC, Tien JH: Drug utilization pattern of Chinese herbal medicines in a general hospital in Taiwan. Pharmacoepidemiol Drug Saf 2005, 14:65 I-657.

18. Chen FP, Kung YY, Chen TJ, Hwang SJ: Demographics and patterns of acupuncture use in the Chinese population: the Taiwan experience. J Compl Altern Med 2006, I 2:379-387.

19. Chen FP, Chen TJ, Kung YY, Chen YC, Chou LF, Chen FJ, Hwang SJ: Use frequency of traditional Chinese medicine in Taiwan. BMC Health Services Res 2007, 7:26-36.

20. National Health Insurance Research Database [http:// www.nhri.org.tw/nhird/date cohort.htm]. (last accessed 27 June 2008)

21. Yang NP, Deng CY, Chou YJ, Chen PQ, Lin CH, Chou P, Chang HJ: Estimated prevalence of osteoporosis from a Nationwide Health Insurance database in Taiwan. Health Policy 2006, 75:329-337.

22. Gardiner P, Graham RE, Legedza AT, Eisenberg DM, Philips RS: Factors associated with dietary supplement use among prescription medication users. Arch Intern Med 2006, 166:1968-1974

23. Gardiner P, Graham R, Legedza AT, Ahn AC, Eisenberg DM, Phillips RS: Factors associated with herbal therapy use by adults in the United States. Altern Ther Health Med 2007, 13:22-29.

24. Department of Health, Taiwan: The list of Taiwan's mountainous and island townships Taipei. (last accessed 27 June 2008) 2002 [http://www.doh.gov.tw/ufile/doc/910201 1 .doc].

25. Bureau of National Health Insurance, Taiwan: 2001 National Health Insurance Annual Statistical Report. Taipei 2002.

26. SAS Institute Inc: SAS® 9.1.3 Language Reference: Dictionary Volume I4. 5th edition. Cary: SAS Institute Inc; 2006.

27. Hedeker D, Gibbons RD: Longitudinal Data Analysis Hoboken: Wiley; 2006.

28. Diggle P, Heagerty P, Liang KY, Zeger SL: Analysis of Longitudinal Dato 2nd edition. Oxford: Oxford University Press; 2002.

29. Wolsko PM, Eisenberg DM, Davis RB, Ettner SL, Philips RS: Insurance coverage, medical conditions, and visits to alternative medicine providers - results of a national survey. Arch Intern Med 2002, 162:28I-287.
30. Ma GX: Between two worlds: the use of traditional and Western health services by Chinese immigrants. J Commun Health 1999, 24:42 I-437.

31. Druss BG, Rosenheck RA: Association between use of unconventional therapies and conventional medical services. JAMA 1999, 282:65I-656.

32. Cleary-Guida $\mathrm{MB}, \mathrm{Okvat} \mathrm{HA}, \mathrm{Oz} \mathrm{MC}$, Ting $\mathrm{W}$ : A regional survey of health insurance coverage for complementary and alternative medicine: Current status and future ramifications. Altern Complem Med 200I, 7:269-273.

33. Paramore LC: Use of alternative therapies: Estimates from the 1994 Robert Wood Johnson Foundation National Access to Care Survey. J Pain Symptom Manag 1997, 13:83-89.

34. Chan MF, Mok E, Wong YS, Tong TF, Day MC, Tang CK, Wong DH: Attitudes of Hong Kong Chinese to traditional Chinese medicine and Western medicine: survey and cluster analysis. Complement Ther Med 2003, I I:103-9.

35. Oldendick R, Coker AL, Wieland D, Raymond R, Probst JC, Schell BJ, Stoskopf $\mathrm{CH}$ : Population-based survey of complementary and alternative medicine usage, patient satisfaction, and physician involvement. South Med J 2000, 93:375-38I.

36. Chen L, Yang WS, Lee SD, Chang HC, Yeh CL: Utilization of wellbaby care visits provided by Taiwan's National Health Insurance Program. Social Sci Med 2004, 59:1647-1659.

37. Wiles J, Rosenberg M: "Gentle caring experience": Seeking alternative health care in Canada. Health Place 200I, 7:2209-2224.

38. D'Crus A, Wilkinson JM: Reasons for choosing and complying with complementary health care: an in-house study on a South Australian clinic. J Altern Complment Med 2005, I I:I I07-I II 2.

39. Chen TJ, Chou LF, Hwang SJ: Patterns of ambulatory care utilization in Taiwan. BMC Health Serv Res 2006, 6:54-61.

40. Cheng TM: Taiwan's new national health insurance program: genesis and experience so far. Health Affairs 2003, 22:6I-76.

4I. Kessler RC, Davis RB, Foster DF, Van Rompay MI, Walters EE, Wilkey SA, Kaptchuk T], Eisenberg DM: Long-term trends in the use of complementary and alternative medical therapies in the United States. Ann Intern Med 200I, I35:262-268.

42. Kennedy J: Herb and supplement use in the US adult population. Clin Therap 2005, I I: 1847-1858.

43. Yamashita H, Tsukayama $H$, Sugishita $C$ : Popularity of complementary and alternative medicine in Japan. A telephone survey. Compl Ther Med 2002, 10:84-93

44. Napadow V, Kaptchuk T]: Patient characteristics for outpatient acupuncture in Beijing, China. J Altern Complement Med 2004, 10:565-572.

45. Oskam N: Alternative Medicine: Care or Blessing Amsterdam: NIPO; 1988.

46. Thomas K, Fall M, Parry C, Nicholl J: National Survey of Access to Complementary Healthcare via General Practice Sheffield: Medical Care Research Unit of the University of Sheffield; 1995.

\section{Pre-publication history}

The pre-publication history for this paper can be accessed here:

\section{http://www.biomedcentral.com/1472-6963/8/170/pre} pub 